\title{
Cholangiocarcinoma-associated genes identified by integrative analysis of gene expression data
}

\author{
WEI ZHONG ${ }^{1}$, LIANZHI DAI ${ }^{2}$, JING LIU $^{1}$ and SONG ZHOU ${ }^{1}$ \\ ${ }^{1}$ Department of General Surgery; ${ }^{2}$ Medical Department, The Affiliated Southeast Hospital of Xiamen University, \\ Zhangzhou, Fujian 363000, P.R. China
}

Received September 30, 2017; Accepted January 22, 2018

DOI: $10.3892 / \mathrm{mmr} .2018 .8594$

\begin{abstract}
Cholangiocarcinoma (CCA) is characterized by delayed diagnosis and poor survival rate. Research efforts have focused on novel diagnostic technologies for this type of cancer. Transcriptomic microarray technology is a useful research strategy for investigating the molecular properties of CCA. The objective of the present study was to identify candidate biomarkers with high potential for clinical application in CCA using a meta-analysis-based approach. Gene expression profiles of CCA were downloaded from the Gene Expression Omnibus database for integrated analysis. All differentially expressed genes (DEGs) were analyzed by Gene Ontology (GO) enrichment analysis and Kyoto Encyclopedia of Genes and Genomes (KEGG) pathway enrichment. Protein-protein interaction (PPI) networks were further constructed, hub proteins were identified and functional modules were extracted. Following integrated analysis of the seven eligible datasets (428 cases and 46 controls), a set of 1,080 DEGs was identified, including 710 upregulated and 370 downregulated genes. Functional enrichment analysis demonstrated that 'chromosome organization' was a significantly enriched GO term in the biological process category. 'DNA replication', 'influenza A', and 'lysosome' were the top three significantly enriched KEGG pathways. Furthermore, PPI network analysis indicated that the significant hub proteins were histone deacetylase 1, cullin-associated NEDD8-dissociated protein 1, ubiquitin D, early growth response protein 1 and glycogen synthase kinase $3 \beta$. The majority of these proteins are involved in CCA. These results provided a set of targets that may help researchers to clarify further the underlying mechanisms of CCA tumorigenesis.
\end{abstract}

Correspondence to: Professor Song Zhou, Department of General Surgery, The Affiliated Southeast Hospital of Xiamen University, 269 Zhanghua Middle Road, Zhangzhou, Fujian 363000, P.R. China E-mail: 175yyptwk@sina.com

Key words: cholangiocarcinoma, microarray, integrative analysis, differentially expressed genes

\section{Introduction}

Cholangiocarcinoma (CCA) is the most common biliary malignancy and the second most common hepatic malignancy, following hepatocellular carcinoma (1). CCA accounts for $10-25 \%$ of primary liver carcinomas (2). CCA may be classified as intrahepatic (ICC), perihilar or distal CCA depending on the anatomical location (3). CCA is more prevalent in Asia compared with western countries. This is primarily attributed to the increased prevalence of established risk factors, including parasitic infections, bile duct cysts and hepatolithiasis (2). However, the incidence of ICC in the USA has increased from 0.44 to 1.18 cases $/ 100,000$ over the past three decades (4). The majority of CCA cases have the characteristics of insidious early and atypical clinical symptoms, rapid progression and poor prognosis. Surgery is the only curative treatment for patients with CCA; however, 50-95\% of cases are not surgical candidates (5). The current 5-year survival rate for CCA following surgery and chemotherapy is $<20 \%$ (6). In addition, CCA is difficult to diagnose, and existing CCA classification systems do not provide insights into the mechanisms of CCA tumorigenesis or potential targets for therapy (7). Therefore, a better understanding of the biology and molecular pathogenesis of CCA may provide the basis to target these markers for tumor prevention or therapy.

Genomic profiling studies have highlighted differing patterns of CCA, helping to stratify patients for targeted therapies (8). Previous studies have investigated the roles of genetic, epigenetic and transcriptomic alterations, in tumor suppressor genes and oncogenes, in the pathogenesis of CCA. Using integrative molecular analysis, Sia et al (9) described two distinct gene signature classes: A proliferation and an inflammatory class. The proliferation class has specific copy number alterations, activation of oncogenic pathways, and is associated with worse outcome. Based on microarray analysis, Jusakul et al (7) additionally revealed four distinct clusters characterized by different clinical features and genomic alterations. These previous results exemplify how genetics, epigenetics and environmental carcinogens may interplay across different geographies to generate distinct molecular subtypes of cancer. In addition, there are studies comparing gene expression profiles between ICC and hepatocellular carcinoma (HCC), in order to identify differences in their carcinogenic mechanisms $(10,11)$. These studies identified 
genetic alterations in CCA that potentially render early diagnosis and precision treatment a possibility.

However, relatively small sample sizes, and differences in control design and platforms, has led to inconsistencies in terms of the identified genes. Additionally, certain studies have used HCC tissue as a control to identify differentially expressed genes (DEGs) in ICC $(10,11)$. All these aspects increase the heterogeneity of the results. Therefore, an integrated analysis of multiple microarray studies may be helpful to define common DEGs and provide additional evidence for understanding the regulatory mechanism of CCA.

In the present study, an integrated analysis to identify DEGs between CCA and non-tumor tissues was performed by integrating gene expression files in the Gene Expression Omnibus (GEO) database using the web-based tool, NetworkAnalyst. The protein-protein interaction (PPI) network of these genes was subsequently constructed and visualized. In addition, significantly enriched functions of these DEGs were screened and analyzed to identify CCA-associated biological processes and pathways.

\section{Materials and methods}

Dataset collection and data processing. Gene expression profiles of CCAs were obtained from the GEO database (www.ncbi.nlm.nih.gov/geo). The following key words were used: 'Homo sapiens' and 'cholangiocarcinoma'. Datasets containing gene expression profiles of CCA and non-tumor tissues or cultured cells were included in the present study. Studies with a sample number $<10$ were excluded. A total of seven datasets were included in this systematic review. The GEO IDs of these seven datasets were GSE26566, GSE32225, GSE32879, GSE89749, GSE22633, GSE45001 and GSE57555 (Table I) (7,9,11-15).

Processed data in matrix form were collected from each qualifying microarray study. A global meta-analysis for identifying DEGs in CCA was conducted using the rank product algorithm (RankProd package in R statistical software; www.r-project. org) implemented in the web-based tool NetworkAnalyst (integrative meta-analysis of expression data; www.networkanalyst.ca) $(16,17)$. Normalized gene expression datasets were uploaded into NetworkAnalyst. The datasets were subsequently processed and annotated to adjust the data format and class labels to a consistent style. Following an integrity check, the random effects model was used to calculate the pooled effect size according to the result of Cochran's Q tests (16).

Functional enrichment analysis of DEGs. The Database for Annotation, Visualization and Integrated Discovery (DAVID; david.abcc.ncifcrf.gov/knowledgebase) $(18,19)$ is a comprehensive set of functional annotation tools. In the present study, gene ontology (GO) enrichment analysis (including biological process, cellular component and molecular function categories) and Kyoto Encyclopedia of Genes and Genomes (KEGG) pathway enrichment analysis for DEGs was performed using the DAVID tool. A P-value of 0.05 was selected as the cutoff criterion.

Construction of PPI network. Network analyst uses a comprehensive, high-quality PPI database downloaded from
InnateDB (20), which is part of the International Molecular Exchange (IMEx) consortium (21). It additionally contains manually curated protein interaction data from published literature and experimental data from a number of PPI databases, including IntAct (22), MINT (23), DIP (24), BIND (25) and BioGRID (26). To construct the gene coexpression network of the DEGs, the DEGs were mapped on the protein interaction network tool in NetworkAnalyst. As the total nodes were $>2,000$, the network was switched to zero-order interactions, which composed only of the seed nodes and the edges that interconnect them (16). Visualization and functional analyses were performed. Furthermore, functional modules from the PPI network in NetworkAnalyst were identified and extracted, using enrichment analysis.

\section{Results}

Data processing and DEG identification. From the GEO database of the National Center for Biotechnology Information, seven GEO datasets associated with CCA that met our criteria for meta-analysis were extracted (Table I). Among these datasets, a total of 428 CCA cases and 46 controls were included in the integrated analysis.

An overview of the meta-analysis approach is outlined in Fig. 1. The meta-analysis page of the NetworkAnalyst website presents five common approaches for meta-analysis. The present study was performed based on combining effect sizes. According to the result of Cochran's Q test (data not shown), the random effects model was chosen for statistical meta-analysis. DEGs with $\mathrm{P}<0.05$ were selected. A total of 12,081 genes were identified by integrated analysis, and 1,080 DEGs were identified from this meta-analysis, including 710 upregulated and 370 downregulated genes. The 10 most significantly upregulated genes $(\mathrm{P}<0.05)$ are secreted phosphoprotein 1 (SPP1), matrix metallopeptidase 11 (MMP11), collagen type I $\alpha 1$ chain (COL1A1), thymosin $\beta 10$ (TMSB10), agrin (AGRN), collagen type IV $\alpha 1$ chain (COL4A1), Collagen type $\mathrm{X} \alpha 1$ chain (COL10A1), minichromosome maintenance complex component 3 (MCM3), collagen type IV $\alpha 2$ chain (COL4A2) and solute carrier family 39 member 1 (SLC39A1; Table IIA). The 10 most significantly downregulated genes $(\mathrm{P}<0.05)$ are FXYD domain containing ion transport regulator 1 (FXYD1), cytochrome P450 family 2 subfamily A member 13 (CYP2A13), cystathionine gamma-lyase (CTH), apolipoprotein F (APOF), ornithine carbamoyltransferase (OTC), hydroxyacid oxidase 2 (HAO2), glycine-N-acyltransferase (GLYAT), phosphoenolpyruvate carboxykinase 2 (PCK2), microsomal triglyceride transfer protein (MTTP) and cytochrome P450 family 4 subfamily A member 22 (CYP4A22; Table IIB). The heat map of the top 100 upregulated and downregulated DEGs is presented in Fig. 2.

Functional enrichment analysis. Functional enrichment analysis was performed to further study these DEGs. Following GO enrichment analysis, three categories (biological process, cellular component and molecular function) were detected using the DAVID database. The 10 most significantly enriched terms $(\mathrm{P}<0.05)$ in each category are presented in Fig. 3. KEGG pathway analysis revealed 23 significantly enriched pathways, with 'DNA replication' being the most significantly enriched 
Table I. Characteristics of the individual studies for integrated analysis.

\begin{tabular}{|c|c|c|c|c|c|}
\hline \multirow[b]{2}{*}{ Author, year } & \multirow[b]{2}{*}{ Datasets } & \multirow[b]{2}{*}{ Platforms } & \multicolumn{2}{|c|}{ Sample type } & \multirow[b]{2}{*}{ (Refs.) } \\
\hline & & & CCA, no. & Control, no. & \\
\hline Jusakul et al, 2017 & GSE89749 & $\begin{array}{l}\text { Illumina HumanHT-12 V4.0 } \\
\text { expression beadchip }\end{array}$ & 118 & 2 & (7) \\
\hline Sia et al, 2013 & GSE32225 & $\begin{array}{l}\text { Illumina HumanRef-8 } \\
\text { WG-DASL v3.0 }\end{array}$ & 149 & 6 & (9) \\
\hline Murakami et al, 2015 & GSE57555 & $\begin{array}{l}\text { Agilent-039494 SurePrint G3 } \\
\text { Human GE v2 8x60K Microarray }\end{array}$ & 11 & 11 & (11) \\
\hline Andersen et al, 2012 & GSE26566 & $\begin{array}{l}\text { Illumina humanRef- } 8 \text { v } 2.0 \\
\text { expression beadchip }\end{array}$ & 104 & 6 & (12) \\
\hline Oishi et al, 2012 & GSE32879 & $\begin{array}{l}\text { Affymetrix Human Gene } 1.0 \\
\text { ST Array }\end{array}$ & 16 & 7 & (13) \\
\hline Seol et al, 2011 & GSE22633 & $\begin{array}{l}\text { Illumina human- } 6 \mathrm{v} 2.0 \\
\text { expression beadchip }\end{array}$ & 20 & 4 & (14) \\
\hline Sulpice et al, 2016 & GSE45001 & $\begin{array}{l}\text { Agilent-028004 SurePrint G3 } \\
\text { Human GE 8x60K Microarray }\end{array}$ & 10 & 10 & (15) \\
\hline
\end{tabular}

CCA, cholangiocarcinoma.

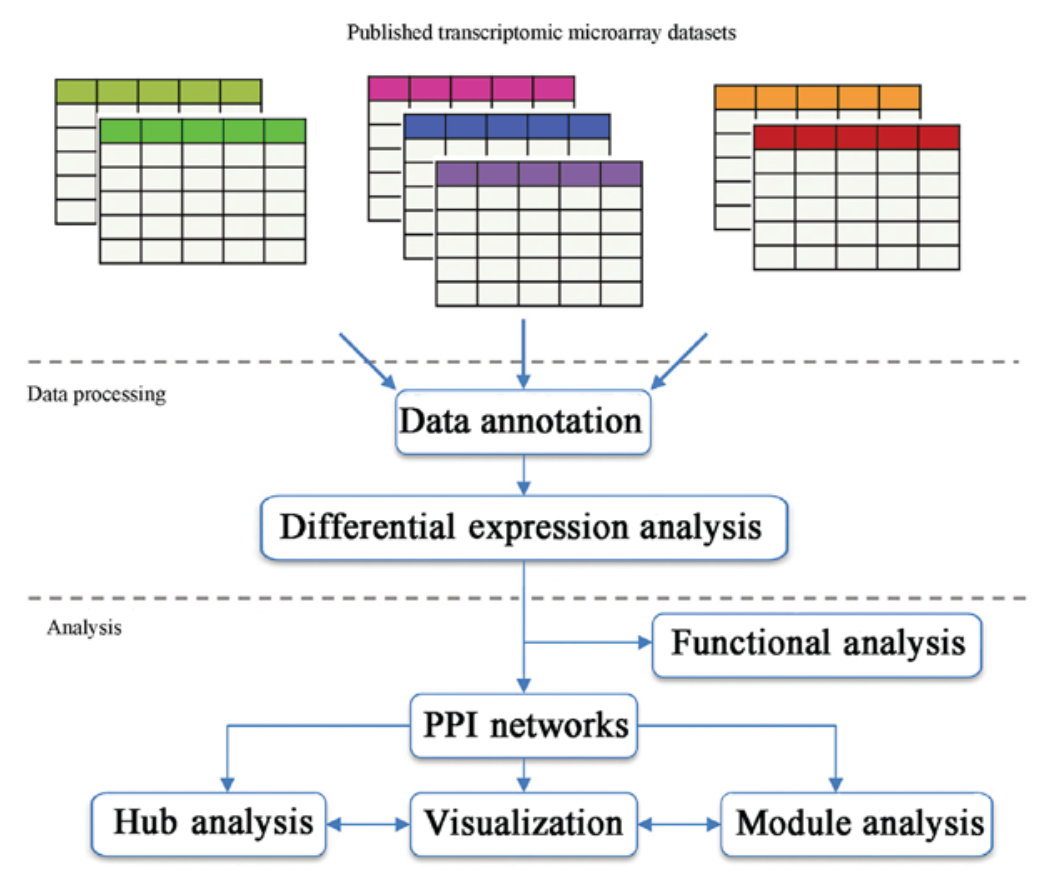

Figure 1. An overview of the meta-analysis approach. A total of seven published transcriptomic microarray datasets were obtained and uploaded to NetworkAnalyst. Following annotation and normalization, the summary-level data (effect size) were extracted and integrated to identify significant differentially expressed genes. Finally, the selected genes were presented within functional enrichment analysis and PPI network analysis. PPI network visualization, hub and module analysis were further performed using Network Analyst. PPI, protein-protein interaction.

pathway. In addition, 'influenza A' and 'lysosome' were also significantly enriched pathways (Fig. 4).

PPI network construction. Based on the IMEx database, the PPI network of the DEGs was constructed using NetworkAnalyst. The interaction network included 471 nodes and 896 edges (Fig. 5). In the PPI network, degrees were defined to determine the number of neighbors a node is directly connected to, and nodes with higher degrees were considered to be hub proteins. The five most significant hub proteins were histone deacetylase 1 (HDAC1; degree=50), cullin-associated NEDD8-dissociated protein 1 (CAND1; degree=47), ubiquitin D (UBD; degree=44), early growth response protein 1 (EGR1; degree=33) and glycogen synthase kinase $3 \beta$ (GSK3B; degree=23). KEGG pathways were subsequently extracted from the PPI network. The five most significantly enriched KEGG pathways in the PPI network were 'influenza A', 'cell cycle', 'pathways in cancer', 'colorectal cancer' and 'T cell 
Table II. Top 10 most significantly up- or downregulated differentially expressed genes.

A, Upregulated genes

\begin{tabular}{llcc}
\hline Entrez ID & Name & Combined ES & P-value \\
\hline 6696 & SPP1 & -2.2097 & 0.014356 \\
4320 & MMP11 & -2.0235 & 0.000592 \\
1277 & COL1A1 & -1.9385 & 0.040954 \\
9168 & TMSB10 & -1.8021 & 0.014024 \\
375790 & AGRN & -1.7029 & 0.008481 \\
1282 & COL4A1 & -1.5809 & 0.004244 \\
1300 & COL10A1 & -1.5545 & 0.000309 \\
4172 & MCM3 & -1.5505 & 0.001245 \\
1284 & COL4A2 & -1.5413 & 0.010102 \\
27173 & SLC39A1 & -1.5064 & 0.015808 \\
\hline
\end{tabular}

B, Downregulated genes

\begin{tabular}{llcc}
\hline Entrez ID & Name & Combined ES & P-value \\
\hline 5348 & FXYD1 & 2.508 & 0.009848 \\
1553 & CYP2A13 & 2.5026 & 0.029454 \\
1491 & CTH & 2.4578 & 0.000691 \\
319 & APOF & 2.4369 & 0.048764 \\
5009 & OTC & 2.4193 & 0.039134 \\
51179 & HAO2 & 2.4088 & 0.040748 \\
10249 & GLYAT & 2.3862 & 0.037987 \\
5106 & PCK2 & 2.3849 & 0.002871 \\
4547 & MTTP & 2.3174 & 0.038962 \\
284541 & CYP4A22 & 2.2502 & 0.013350 \\
\hline
\end{tabular}

DEG, differentially expressed gene; ES, effect size.

receptor signaling pathway' (Fig. 6). Numerous hub genes were associated with these pathways.

\section{Discussion}

CCA is the most common primary malignancy of the biliary tract. The prognosis of this malignancy is dismal owing to its silent clinical character, difficulties in early diagnosis and limited therapeutic approaches; median survival is less than 24 months (27). There are a number of established risk factors for the development of CCA, although the majority of patients have no identifiable risks (2). Therefore, the identification of novel tumor biomarkers for the early diagnosis and effective treatment of patients with CCA is an important future direction. The transcriptional regulatory network, screened and analyzed with advanced technologies including transcriptomic and proteomic analysis, may be informative to understand the underlying regulatory mechanisms and provide additional evidence for therapeutic applications.

In the present study, a total of 1,080 DEGs were identified based on an integrated analysis. Through a PubMed literature search, it was identified that six out of the 10 most upregulated genes have been associated with CCA in biological or clinical experiments: SPP1 (28,29), MMP11 (30), COL1A1 (31), TMSB10 (32), AGRN (33), and COL4A1 (29). By microarray analysis and reverse transcription-quantitative polymerase chain reaction, Hass et al (28) determined that SPP1 is the most overexpressed gene in ICC. Another study demonstrated that SPP1 expression in the stroma of ICC is significantly associated with the overall patient survival (29). A previous study illustrated the role of MMP11 in cancer progression, with positive MMP11 expression in CCA indicating poor prognosis (30). COL1A1 is a component of type I collagen, which has been reported to be involved in tumor invasion and progression. COL1A1 is significantly upregulated in CCA compared with non-tumor tissues (31). Tissue microarray analysis by Sulpice et al (29) demonstrated increased expression of COL4A1 in the stroma of ICC. Abnormal expression of TMSB10 may contribute to the malignant progression of $\mathrm{HCC}$, and high expression of TMSB10 predicts poor prognosis in patients with HCC following hepatectomy (34). High TMSB10 expression is significantly associated with clinicopathological features, poor prognosis, and distant metastases in patients with breast cancer (35). AGRN is a multidomain heparan sulfate proteoglycan, with different modules homologous to domains present in basement membrane proteins. AGRN expression and deposits are increased in CCA compared with HCC and nontumorous livers, which implies multiple roles in the pathogenesis and progression of CCA (33). However, few downregulated DEGs have been reported to be associated with CCA. Certain genes may be associated with CCA indirectly. For example, the metabolic gene $\mathrm{HAO} 2$ is downregulated in $\mathrm{HCC}$, and $\mathrm{HAO} 2$ expression levels are inversely correlated with grading, overall survival and metastatic ability (36). Cystathionine- $\gamma$-lyase expression is regulated by the Wnt pathway at the transcriptional level and is involved in colon cancer (37); it additionally leads to the development of breast cancer in association with the STAT3 signaling pathway (38). The low expression level of these genes may be associated with the development of CCA, although there are no specific experiments investigating these genes in CCA.

The functional mechanisms of these DEGs using GO and KEGG pathway analyses were further investigated. A total of 482 significantly enriched terms in the biological process, 42 in the cellular component and 73 in the molecular function category were identified. All of the 10 most significantly enriched terms in the biological process category are associated with DNA and chromosomes. Active DNA synthesis means proliferation signaling pathways may be activated in CCA, and an activated cell cycle process may be a sign of proliferation or cancer progression. There were 141 DEGs enriched in the cell cycle process, including enhancer of zeste 2 polycomb repressive complex 2 subunit (EZH2), cyclin $\mathrm{C}$ and cyclin $\mathrm{E} 1$. EZH2 expression promotes the progression of CCA cells by regulating the cell cycle and is associated with poor CCA prognosis (39). In the top 10 cell cycle process terms, apart from terms associated with DNA and chromosomes, there were three GO terms associated with extracellular components: 'Extracellular exosome', 'extracellular vesicle' and 'extracellular organelle'. Among the 10 most significantly upregulated DEGs, SPP1, MMP11, 


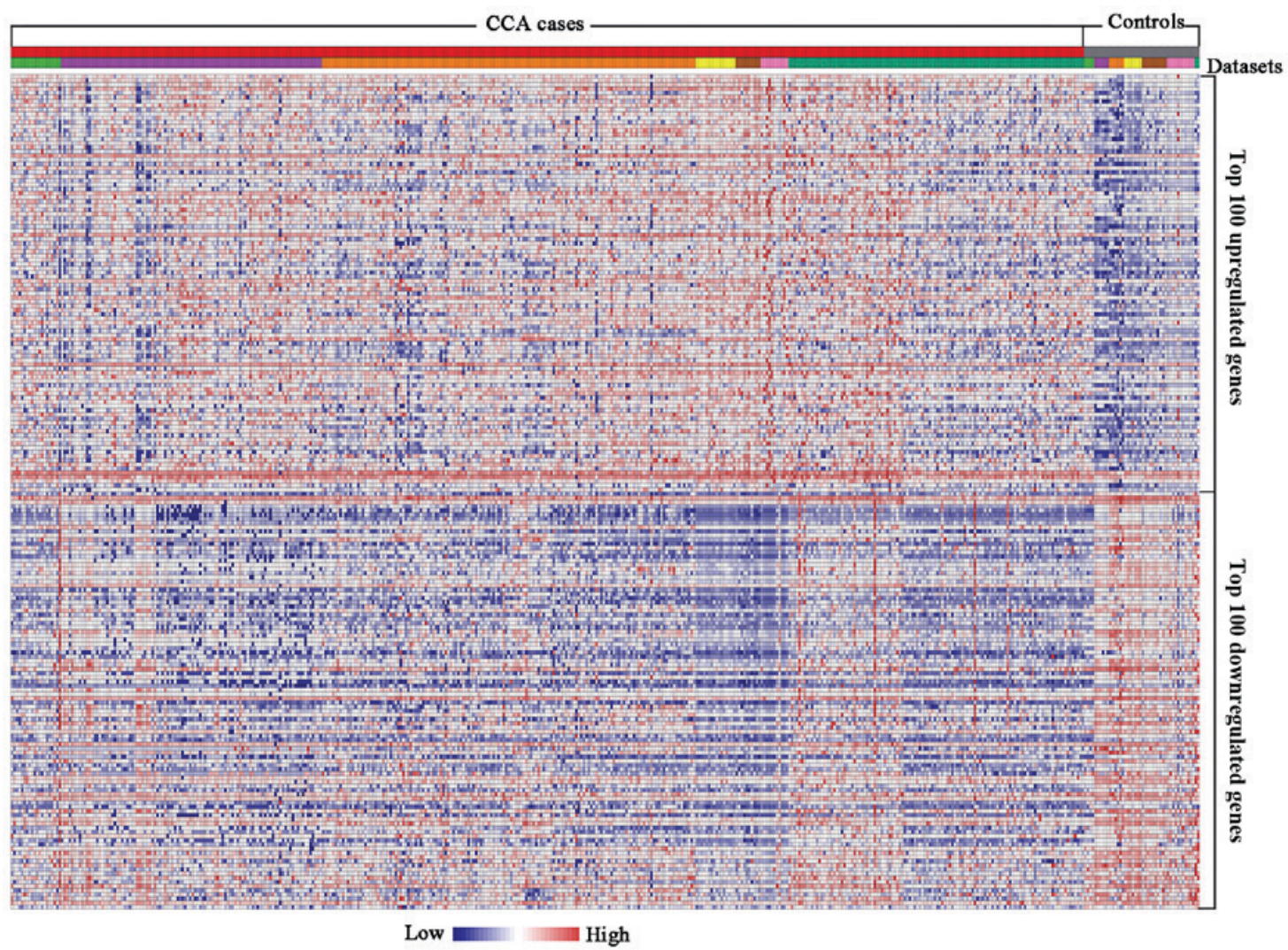

Figure 2. Heat map of differentially expressed genes. Heat maps representing expression patterns for the 100 most upregulated and downregulated genes across different datasets by row-wise comparison. Sample type (the first row): Red indicates the primary tumors of CCA ( $\mathrm{n}=428$ ); grey indicates non-tumor tissue $(\mathrm{n}=46)$. For each gene, red is upregulated and blue is downregulated in the corresponding sample. CCA, cholangiocarcinoma.

GO functional analysis

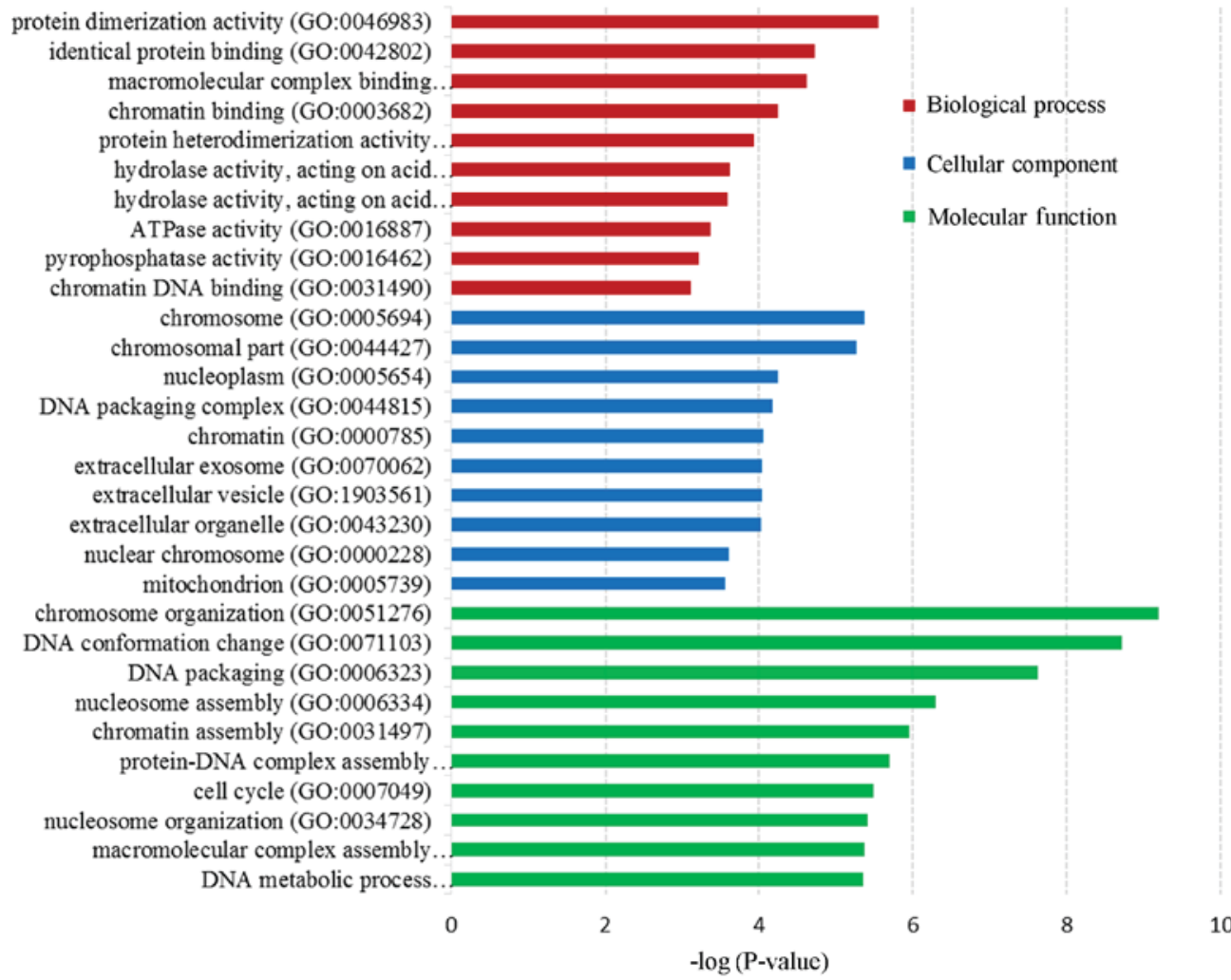

Figure 3. GO functional analysis of DEGs. GO enrichment analysis of DEGs was retrieved using DAVID. The 10 most significantly (P<0.05) enriched GO terms in biological process, molecular function and cellular component branches are presented. All the adjusted statistically significant values of the terms were negative 10-base log transformed. DEGs, differentially expressed genes; GO, gene ontology. 


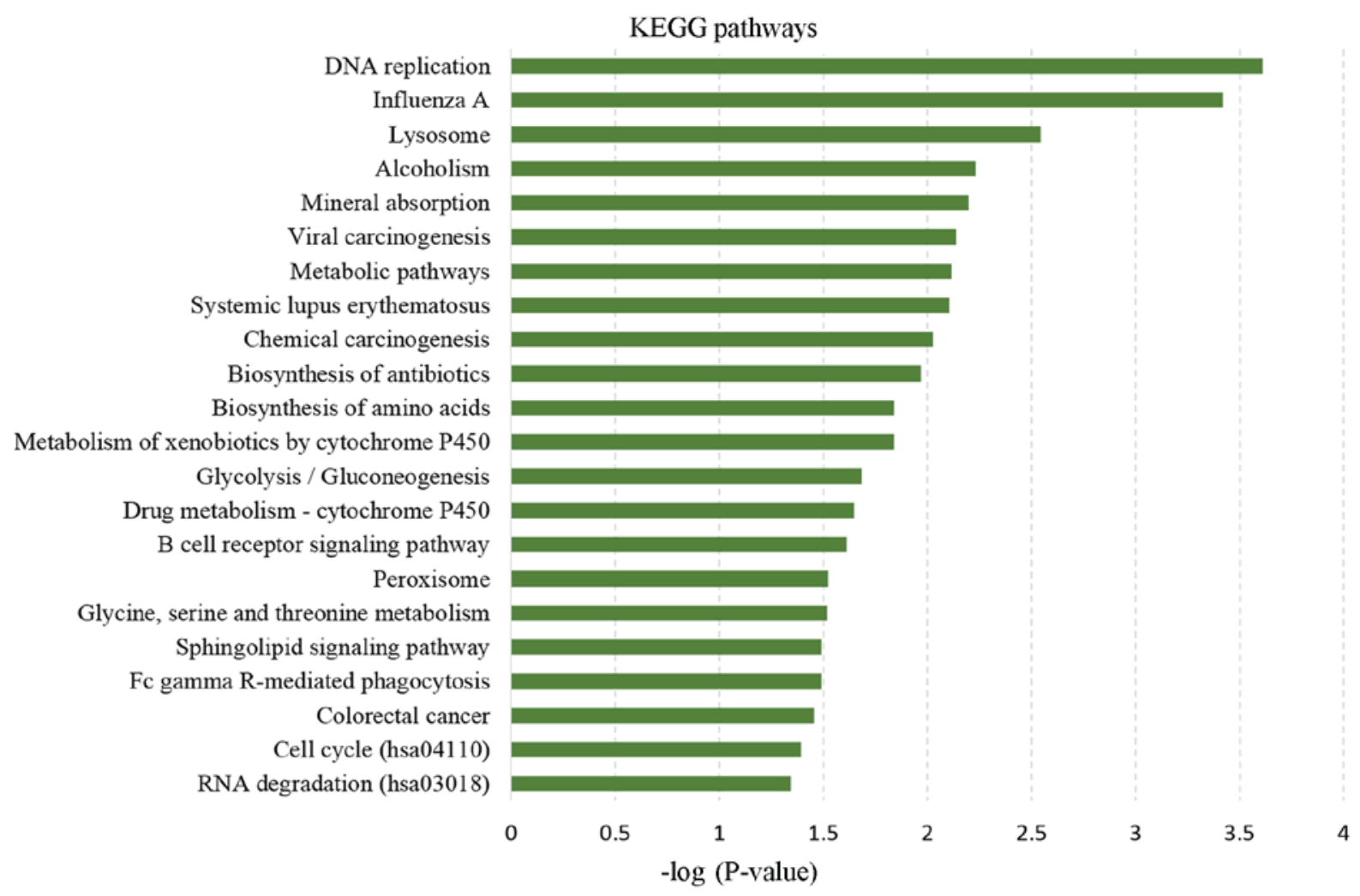

Figure 4. Significantly enriched pathways in KEGG pathway analysis of differentially expressed genes in cholangiocarcinoma. Enriched biological pathways were identified using the Database for Annotation, Visualization and Integrated Discovery. Statistically significant $(\mathrm{P}<0.05)$ categories among the pathways identified in KEGG. The adjusted statistically significant values were negative 10-base log transformed. KEGG, Kyoto Encyclopedia of Genes and Genomes.

COL1A1, COL4A1, COL4A2 and COL10A1 are able to be secreted to the extracellular space $(40,41)$. These results indicated enhanced communication between CCA and the tumor microenvironment. Thus, the tumor microenvironment may be a crucial component governing tumor development and progression and, by targeting it, may be a new direction for CCA treatment (42).

Following KEGG pathway analysis, 22 pathways were identified according to the P-value cut-off. There were five terms, in accordance with the results published by Huang et al (31). Certain pathways, including 'alcoholism' (hsa05034), 'viral carcinogenesis' (hsa05203) and 'colorectal cancer' (hsa05210) have a close association with CCA. Additionally, heavy alcohol use, hepatitis B virus infection, hepatitis $C$ virus infection and inflammatory bowel disease are possible risk factors for CCA (2). Furthermore, a PPI network was established using the NetworkAnalyst visualization tool. PPI network analysis revealed the significant hub proteins, HDAC1, CAND1, UBD, EGR1 and GSK3B. HDACs serve an oncogenic role in the occurrence and development of ICC. Abnormal expression of HDAC1 is significantly associated with lymph node metastasis, high stage carcinoma, vascular invasion and poor prognosis of ICC (43).

It was hypothesized that analyzing multiple datasets may increase the accuracy of the findings, compared with conclusions raised from analyzing a single datasets. In addition, identifying cell markers that are overexpressed in the majority of CCAs may lead to novel drug targets with high specificity. The present results may help to identify combinations of treatments to target various signaling pathways that are altered in CCA. However, certain limitations remain with the present study. Firstly, DEGs were identified via integrated analysis of microarray data. Although differential expression of several of these genes was confirmed by previous biological or clinical research, further in vitro and in vivo validation of these results is required. Secondly, the DEGs identified only represent relative expression levels compared with CCA and non-tumor tissues. Gene mutation and methylation, which are also important for CCA tumorigenesis and progression, were not included in the present analysis. Thirdly, the integrative analysis method used in the present study is based on combining the effect sizes, although there are a number of other algorithms used to identify DEGs, including P-values and gene ranks (15). Different methods may increase the heterogeneity between different published analyses of DEGs. Additionally, the sample cases are much more than the controls (428 vs. 46), and this case-control ratio may be another limitation of the present study.

In summary, the present study identified potential novel markers in the pathogenesis of CCA. In addition, genes consistently differing in expression in CCA were identified through NetworkAnalyst tools. The 10 most significantly upregulated and downregulated genes may serve as potential diagnostic biomarkers. GO annotation and KEGG pathway analysis demonstrated that the identified candidates have a strong association with CCA. Furthermore, a number of novel CCA-associated genes were identified. Further experimental validation is required to fully understand the mechanism of these novel genes in CCA, and to investigate any therapeutic and diagnostic potential these genes may hold. 


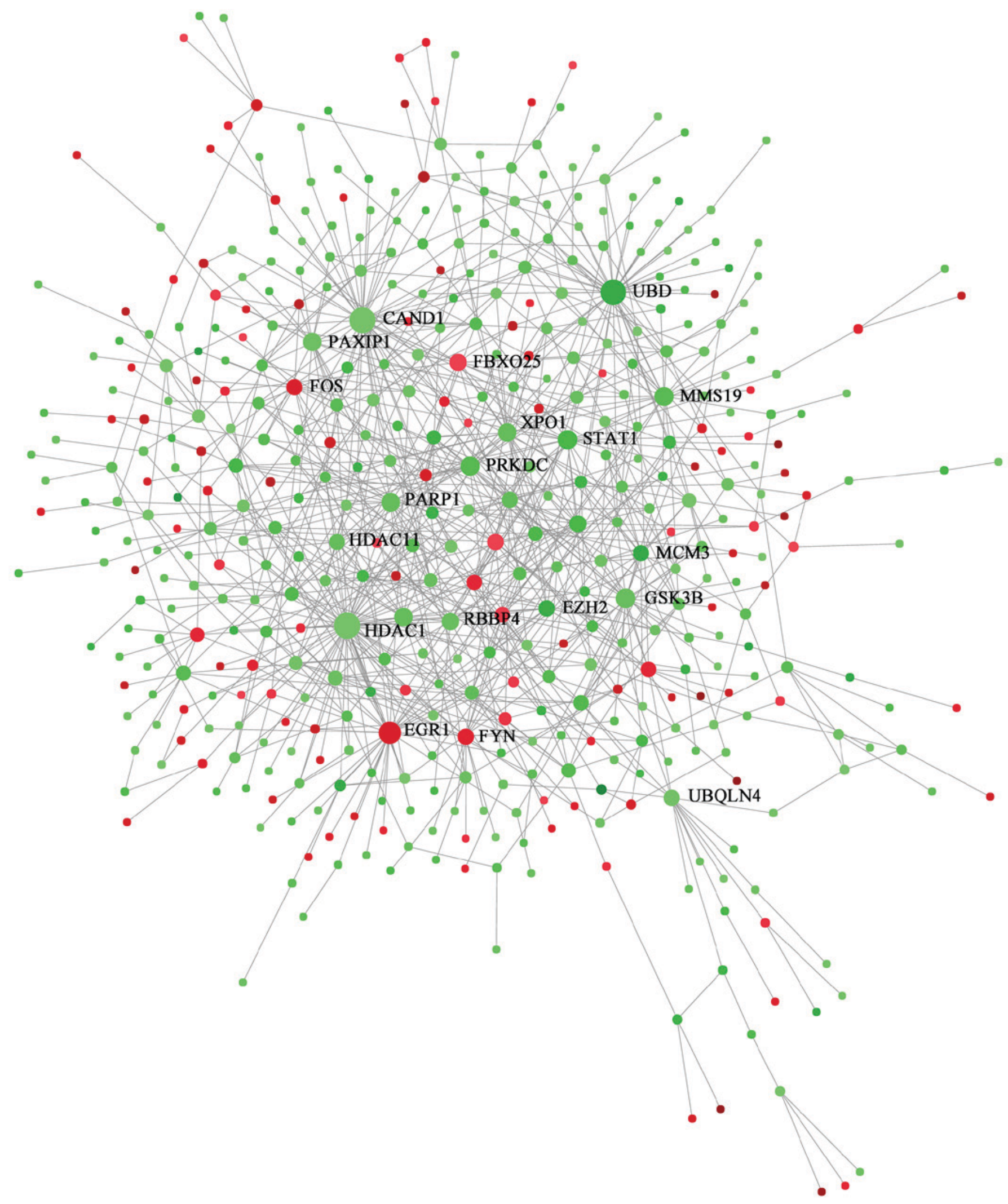

Figure 5. An overview of the PPI network. The PPI network was generated using NetworkAnalyst. Red and green color nodes represent upregulated and downregulated differentially expressed genes, respectively. PPI, protein-protein interaction. 


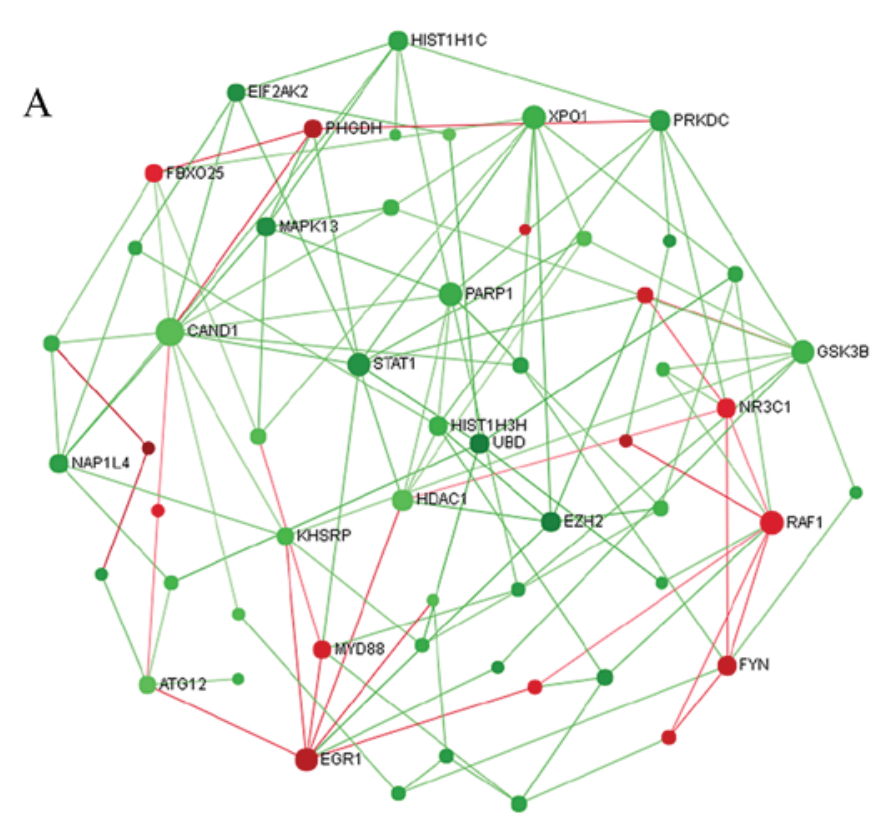

Influenza A
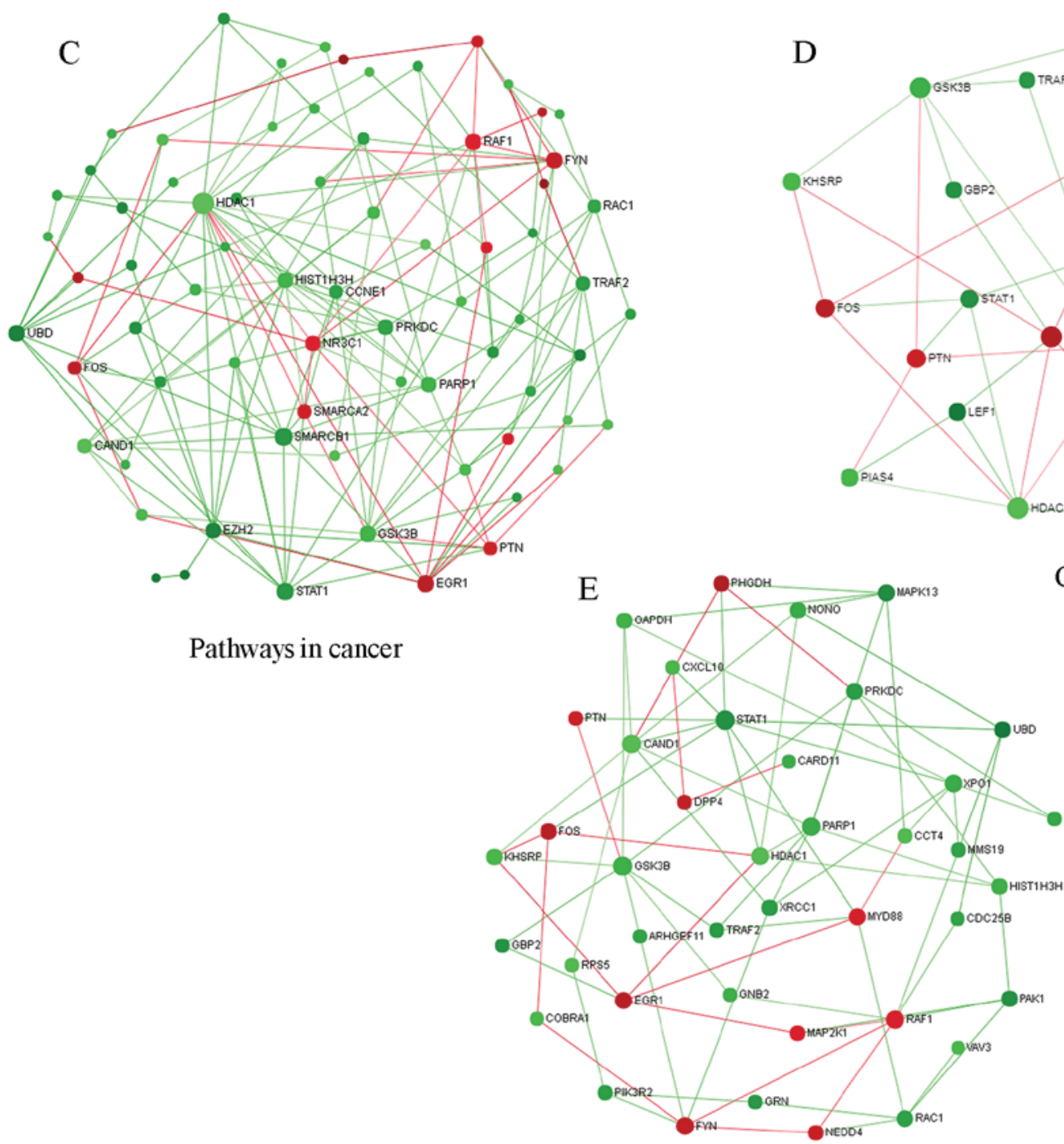

TCR pathway

Pathways in cancer

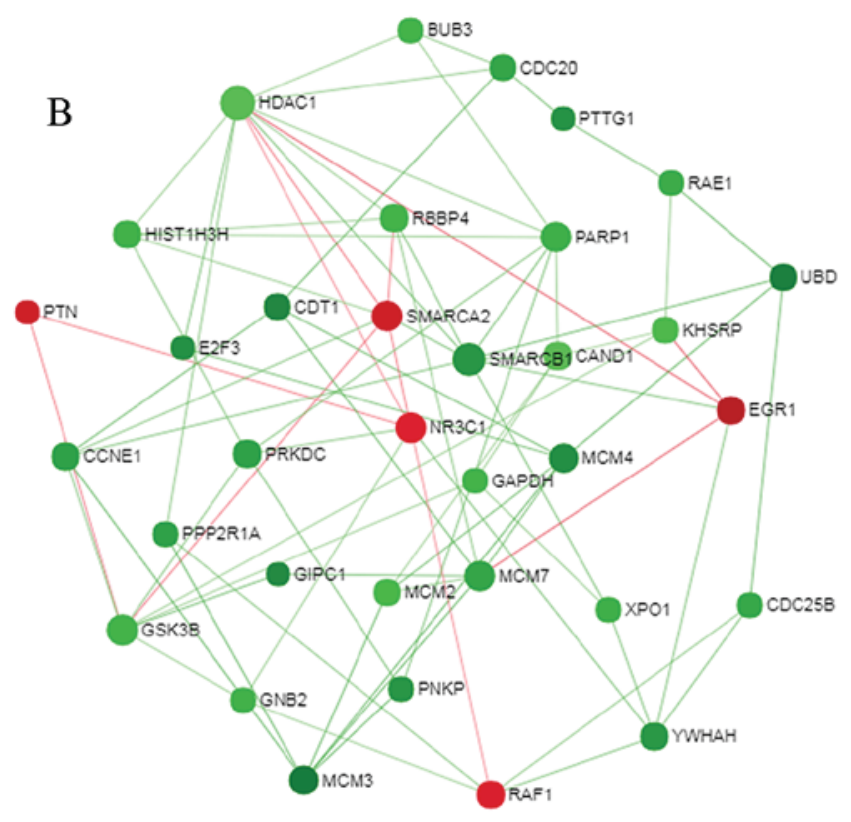

Cell cycle

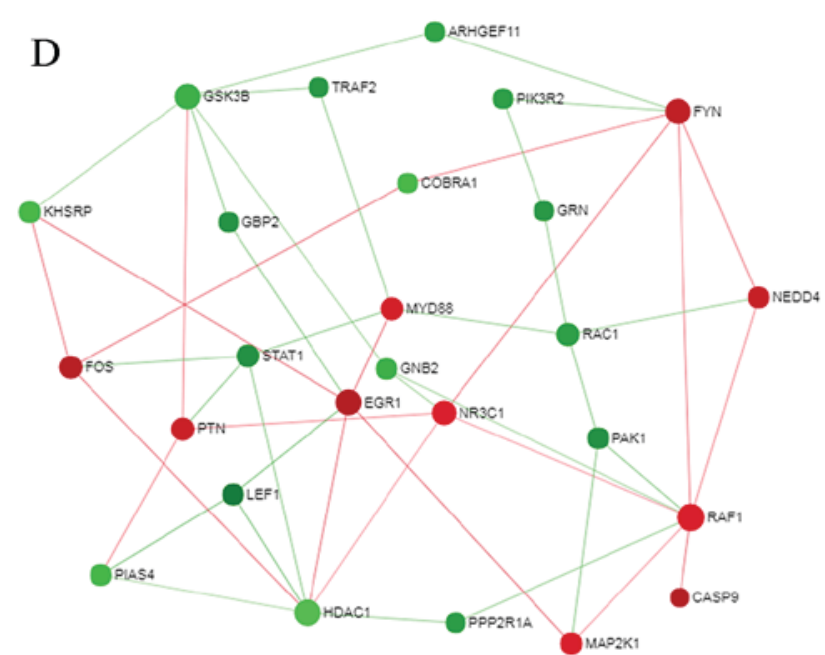

Colorectal cancer

Figure 6. The five most significantly enriched Kyoto Encyclopedia of Genes and Genomes pathways extracted from the protein-protein interaction network. (A) Influenza A; (B) cell cycle; (C) pathways in cancer; (D) colorectal cancer; (E) TCR signaling pathway. Red and green colored nodes represent upregulated and downregulated genes, respectively. TCR, T cell receptor. 


\section{Acknowledgements}

Not applicable.

\section{Funding}

The present study was supported by nursery grants from the 175th Hospital of People's Liberation Army, Zhang Zhou, China (grant no. 16Y006).

\section{Availability of data and materials}

The datasets used and/or analyzed during the current study are available from the corresponding author on reasonable request.

\section{Authors' contributions}

The authors' contributions were as follows: WZ, conception and design, data collection, data analysis and manuscript writing; LD, data collection and data analysis; JL, conception and design and data analysis; SZ, conception and design and final approval of manuscript. All authors read and approved the final manuscript.

\section{Ethics approval and consent to participate}

Not applicable.

\section{Consent for publication}

Not applicable.

\section{Competing interests}

The authors declare that they have no competing interests.

\section{References}

1. de Groen PC, Gores GJ, LaRusso NF, Gunderson LL and Nagorney DM: Biliary tract cancers. N Engl J Med 341: 1368-1378, 1999.

2. Tyson GL and El-Serag HB: Risk factors for cholangiocarcinoma. Hepatology 54: 173-184, 2011.

3. Rizvi S and Gores GJ: Pathogenesis, diagnosis, and management of cholangiocarcinoma. Gastroenterology 145: 1215-1229, 2013.

4. Saha SK, Zhu AX, Fuchs CS and Brooks GA: Forty-year trends in cholangiocarcinoma incidence in the U.S.: Intrahepatic disease on the rise. Oncologist 21: 594-599, 2016

5. Oliveira IS, Kilcoyne A, Everett JM, Mino-Kenudson M, Harisinghani MG and Ganesan K: Cholangiocarcinoma: Classification, diagnosis, staging, imaging features, and management. Abdom Radiol (NY) 42: 1637-1649, 2017.

6. Khuntikeo N, Chamadol N, Yongvanit P, Loilome W, Namwat N, Sithithaworn P, Andrews RH, Petney TN, Promthet S, Thinkhamrop K, et al: Cohort profile: Cholangiocarcinoma screening and care program (CASCAP). BMC Cancer 15: 459, 2015.

7. Jusakul A, Cutcutache I, Yong CH, Lim JQ, Huang MN Padmanabhan N, Nellore V, Kongpetch S, Ng AWT, Ng LM, et al: Whole-genome and epigenomic landscapes of etiologically distinct subtypes of cholangiocarcinoma. Cancer Discov 7: 1116-1135, 2017.

8. Kayhanian H, Smyth EC and Braconi C: Emerging molecular targets and therapy for cholangiocarcinoma. World J Gastrointest Oncol 9: 268-280, 2017.

9. Sia D, Hoshida Y, Villanueva A, Roayaie S, Ferrer J, Tabak B, Peix J, Sole M, Tovar V, Alsinet C, et al: Integrative molecular analysis of intrahepatic cholangiocarcinoma reveals 2 classes that have different outcomes. Gastroenterology 144: 829-840, 2013.
10. Seok JY, Na DC, Woo HG, Roncalli M, Kwon SM, Yoo JE, Ahn EY, Kim GI, Choi JS, Kim YB and Park YN: A fibrous stromal component in hepatocellular carcinoma reveals a cholangiocarcinoma-like gene expression trait and epithelial-mesenchymal transition. Hepatology 55: 1776-1786, 2012.

11. Murakami Y, Kubo S, Tamori A, Itami S, Kawamura E, Iwaisako K, Ikeda K, Kawada N, Ochiya T and Taguchi YH: Comprehensive analysis of transcriptome and metabolome analysis in intrahepatic cholangiocarcinoma and hepatocellular carcinoma. Sci Rep 5: 16294, 2015.

12. Andersen JB, Spee B, Blechacz BR, Avital I, Komuta M, Barbour A, Conner EA, Gillen MC, Roskams T, Roberts LR, et al: Genomic and genetic characterization of cholangiocarcinoma identifies therapeutic targets for tyrosine kinase inhibitors. Gastroenterology 142: 1021-1031.e15, 2012.

13. Oishi N, Kumar MR, Roessler S, Ji J, Forgues M, Budhu A, Zhao X, Andersen JB, Ye QH, Jia HL, et al: Transcriptomic profiling reveals hepatic stem-like gene signatures and interplay of miR-200c and epithelial-mesenchymal transition in intrahepatic cholangiocarcinoma. Hepatology 56: 1792-1803, 2012.

14. Seol MA, Chu IS, Lee MJ, Yu GR, Cui XD, Cho BH, Ahn EK, Leem SH, Kim IH and Kim DG: Genome-wide expression patterns associated with oncogenesis and sarcomatous transdifferentation of cholangiocarcinoma. BMC Cancer 11: 78, 2011.

15. Sulpice L, Desille M, Turlin B, Fautrel A, Boudjema K, Clément B and Coulouarn C: Gene expression profiling of the tumor microenvironment in human intrahepatic cholangiocarcinoma. Genom Data 7: 229-232, 2016.

16. Xia J, Gill EE and Hancock RE: NetworkAnalyst for statistical, visual and network-based meta-analysis of gene expression data. Nat Protoc 10: 823-844, 2015.

17. Xia J, Benner MJ and Hancock RE: NetworkAnalyst-integrative approaches for protein-protein interaction network analysis and visual exploration. Nucleic Acids Res 42 (Web Server Issue): W167-W174, 2014.

18. Huang da W, Sherman BT and Lempicki RA: Systematic and integrative analysis of large gene lists using DAVID bioinformatics resources. Nat Protoc 4: 44-57, 2009.

19. Huang da W, Sherman BT and Lempicki RA: Bioinformatics enrichment tools: Paths toward the comprehensive functional analysis of large gene lists. Nucleic Acids Res 37: 1-13, 2009.

20. Breuer K, Foroushani AK, Laird MR, Chen C, Sribnaia A, Lo R, Winsor GL, Hancock RE, Brinkman FS and Lynn DJ: InnateDB: Systems biology of innate immunity and beyond-recent updates and continuing curation. Nucleic Acids Res 41 (Database Issue): D1228-D1233, 2013.

21. Orchard S, Kerrien S, Abbani S, Aranda B, Bhate J, Bidwell S, Bridge A, Briganti L, Brinkman FS, Cesareni G, et al: Protein interaction data curation: The International Molecular Exchange (IMEx) consortium. Nat Methods 9: 345-350, 2012.

22. Hermjakob H, Montecchi-Palazzi L, Lewington C, Mudali S, Kerrien S, Orchard S, Vingron M, Roechert B, Roepstorff P, Valencia A, et al: IntAct: An open source molecular interaction database. Nucleic Acids Res 32 (Database Issue): D452-D455, 2004.

23. Licata L, Briganti L, Peluso D, Perfetto L, Iannuccelli M, Galeota E, Sacco F, Palma A, Nardozza AP, Santonico E, et al: MINT, the molecular interaction database: 2012 update. Nucleic Acids Res 40 (Database Issue): D857-D861, 2012.

24. Salwinski L, Miller CS, Smith AJ, Pettit FK, Bowie JU and Eisenberg D: The database of interacting proteins: 2004 update. Nucleic Acids Res 32 (Database Issue): D449-D451, 2004.

25. Isserlin R,El-Badrawi RA and Bader GD: The biomolecular interaction network database in PSI-MI 2.5. Database (Oxford) 2011: baq037, 2011.

26. Chatr-Aryamontri A, Breitkreutz BJ, Oughtred R, Boucher L, Heinicke S, Chen D, Stark C, Breitkreutz A, Kolas N, O'Donnell L, et al: The BioGRID interaction database: 2015 update. Nucleic Acids Res 43 (Database Issue): D470-D478, 2015.

27. Blechacz B, Komuta M, Roskams T and Gores GJ: Clinical diagnosis and staging of cholangiocarcinoma. Nat Rev Gastroenterol Hepatol 8: 512-522, 2011.

28. Hass HG, Nehls O, Jobst J, Frilling A, Vogel U and Kaiser S: Identification of osteopontin as the most consistently overexpressed gene in intrahepatic cholangiocarcinoma: Detection by oligonucleotide microarray and real-time PCR analysis. World $\mathbf{J}$ Gastroenterol 14: 2501-2510, 2008 
29. Sulpice L, Rayar M, Desille M, Turlin B, Fautrel A, Boucher E, Llamas-Gutierrez F, Meunier B, Boudjema K, Clément B and Coulouarn C: Molecular profiling of stroma identifies osteopontin as an independent predictor of poor prognosis in intrahepatic cholangiocarcinoma. Hepatology 58: 1992-2000, 2013.

30. Tongtawee T, Kaewpitoon SJ, Loyd R, Chanvitan S, Leelawat K, Praditpol N, Jujinda S and Kaewpitoon N: High expression of matrix metalloproteinase-11 indicates poor prognosis in human cholangiocarcinoma. Asian Pac J Cancer Prev 16: 3697-3701, 2015.

31. Huang QX, Cui JY, Ma H, Jia XM, Huang FL and Jiang LX: Screening of potential biomarkers for cholangiocarcinoma by integrated analysis of microarray data sets. Cancer Gene Ther 23 : 48-53, 2016.

32. Sawanyawisuth K, Wongkham C, Araki N, Zhao Q, Riggins GJ and Wongkham S: Serial analysis of gene expression reveals promising therapeutic targets for liver fluke-associated cholangiocarcinoma. Asian Pac J Cancer Prev 13 (Suppl): S89-S93, 2012.

33. Batmunkh E, Tátrai P, Szabó E, Lódi C, Holczbauer A, Páska C, Kupcsulik P, Kiss A, Schaff Z and Kovalszky I: Comparison of the expression of agrin, a basement membrane heparan sulfate proteoglycan, in cholangiocarcinoma and hepatocellular carcinoma. Hum Pathol 38: 1508-1515, 2007.

34. Wang H, Jiang S, Zhang Y, Pan K, Xia J and Chen M: High expression of thymosin beta 10 predicts poor prognosis for hepatocellular carcinoma after hepatectomy. World J Surg Oncol 12: 226, 2014.

35. Zhang X, Ren D, Guo L, Wang L, Wu S, Lin C, Ye L, Zhu J, Li J, Song L, et al: Thymosin beta 10 is a key regulator of tumorigenesis and metastasis and a novel serum marker in breast cancer Breast Cancer Res 19: 15, 2017.

36. Mattu S, Fornari F, Quagliata L, Perra A, Angioni MM, Petrelli A, Menegon S, Morandi A, Chiarugi P,Ledda-Columbano GM, et al: The metabolic gene HAO2 is downregulated in hepatocellular carcinoma and predicts metastasis and poor survival J Hepatol 64: 891-898, 2016.
37. Fan K, Li N, Qi J, Yin P, Zhao C, Wang L, Li Z and Zha X: Wnt/ $\beta$-catenin signaling induces the transcription of cystathionine- $\gamma$-lyase, a stimulator of tumor in colon cancer. Cell Signal 26: 2801-2808, 2014

38. You J, Shi X, Liang H, Ye J, Wang L, Han H, Fang H, Kang W and Wang T: Cystathionine- $\gamma$-lyase promotes process of breast cancer in association with STAT3 signaling pathway. Oncotarget 8: 65677-65686, 2017.

39. Nakagawa S, Okabe H, Sakamoto Y, Hayashi H, Hashimoto D, Yokoyama N, Sakamoto K, Kuroki H, Mima K, Nitta H, et al: Enhancer of zeste homolog 2 (EZH2) promotes progression of cholangiocarcinoma cells by regulating cell cycle and apoptosis. Ann Surg Oncol 20 (Suppl 3): S667-S675, 2013.

40. Giussani M, Merlino G, Cappelletti V, Tagliabue E and Daidone MG: Tumor-extracellular matrix interactions: Identification of tools associated with breast cancer progression. Semin Cancer Biol 35: 3-10, 2015.

41. Kuo DS, Labelle-Dumais C and Gould DB: COL4A1 and COL4A2 mutations and disease: Insights into pathogenic mechanisms and potential therapeutic targets. Hum Mol Genet 21: R97-R110, 2012.

42. Razumilava N and Gores GJ: Cholangiocarcinoma. Lancet 383: 2168-2179, 2014.

43. Morine Y, Shimada M, Iwahashi S, Utsunomiya T, Imura S, Ikemoto T, Mori H, Hanaoka J and Miyake H: Role of histone deacetylase expression in intrahepatic cholangiocarcinoma. Surgery 151: 412-419, 2012.

(i) (8) This work is licensed under a Creative Commons

cc. At No ND Atribution-NonCommercial-NoDerivatives 4.0 International (CC BY-NC-ND 4.0) License. 